\title{
Formation of a non-magnetic, odd- denominator fractional quantized conductance in a quasi-one-dimensional electron system
}

Cite as: Appl. Phys. Lett. 115, 123104 (2019); https://doi.org/10.1063/1.5121147

Submitted: 22 July 2019 . Accepted: 27 August 2019 . Published Online: 18 September 2019

S. Kumar (D), M. Pepper (D), D. Ritchie, I. Farrer (D), and H. Montagu
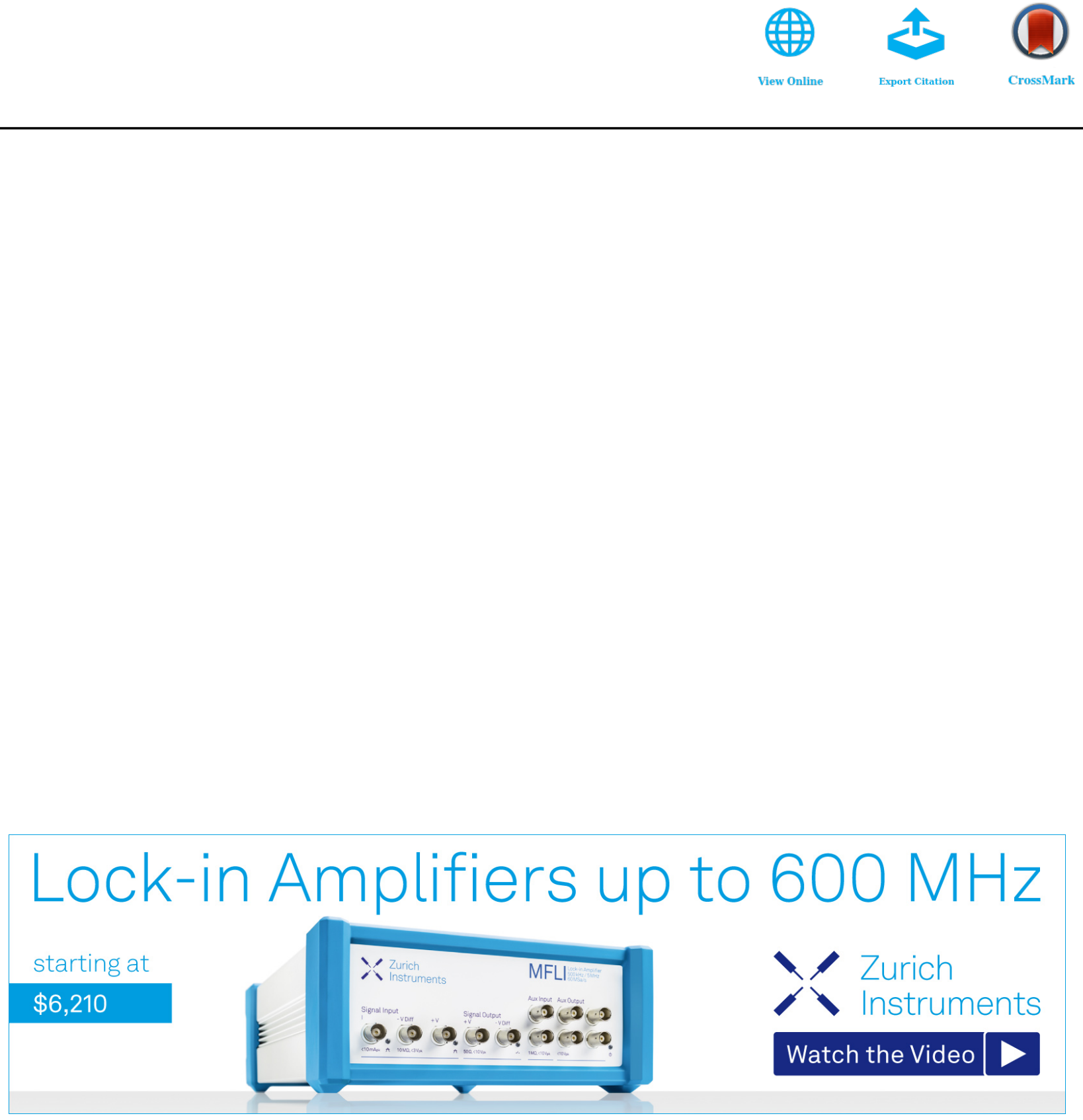


\title{
Formation of a non-magnetic, odd-denominator fractional quantized conductance in a quasi-one-dimensional electron system
}

Cite as: Appl. Phys. Lett. 115, 123104 (2019); doi: 10.1063/1.5121147

Submitted: 22 July 2019 . Accepted: 27 August 2019 .

Published Online: 18 September 2019

S. Kumar, ${ }^{1,2, a)}$ (D) M. Pepper, ${ }^{1,2}$ (D) D. Ritchie, ${ }^{3}$ I. Farrer, ${ }^{3,4}$ (D) and H. Montagu ${ }^{1,2}$

\begin{abstract}
AFFILIATIONS
${ }^{7}$ Department of Electronic and Electrical Engineering, University College London, Torrington Place, London WC1E 7JE, United Kingdom

${ }^{2}$ London Centre for Nanotechnology, 17-19 Gordon Street, London WC1H OAH, United Kingdom

${ }^{3}$ Cavendish Laboratory, J.J. Thomson Avenue, Cambridge CB3 OHE, United Kingdom

${ }^{4}$ Department of Electronic and Electrical Engineering, University of Sheffield, Mappin Street, Sheffield S1 3JD, United Kingdom

a) Author to whom correspondence should be addressed: sanjeev.kumar@ucl.ac.uk
\end{abstract}

\begin{abstract}
We have investigated the transport properties of a two-dimensional electron gas confined to one dimension by voltages applied to a pair of split gates and an additional top gate deposited on the surface of the GaAs/AlGaAs heterostructure. At low carrier concentrations, $<5 \times 10^{10} \mathrm{~cm}^{-2}$, in a wide $1 \mathrm{D}$ channel $(700 \mathrm{~nm})$, with an asymmetric confinement potential, the conductance plateaux in units of $\mathrm{e}^{2} / \mathrm{h}$ at 1 and $2 / 3$ were observed. In the presence of a fixed in-plane magnetic field of $10 \mathrm{~T}$, additional plateaux at 2/5 and 2/7 appeared with increasing asymmetry in confinement potential. The appearance of odd-denominator fractional states seems to originate from the zigzag formed when electrons are allowed to relax in the second dimension at the boundary of the $1 \mathrm{D}-2 \mathrm{D}$ transition.
\end{abstract}

Published under license by AIP Publishing. https://doi.org/10.1063/1.5121147

The observation of the quantized conductance of a onedimensional (1D) electron system in units of $2 \mathrm{e}^{2} / \mathrm{h}$, where e is the charge of electron and $\mathrm{h}$ is the Planck constant, stimulated the field of less than two-dimensional semiconductors. ${ }^{1-3}$ This has now evolved significantly, and a variety of fundamental quantum effects have been reported including the $0.7\left(2 \mathrm{e}^{2} / \mathrm{h}\right)$ conductance anomaly, spinincoherent transport, Wigner crystallization and its detection, and many other associated effects. ${ }^{4-9}$ Of these, the $0.7\left(2 \mathrm{e}^{2} / \mathrm{h}\right)$ conductance anomaly, a plateau appearing just below the first conductance plateau, has been extensively investigated with considerable discussion on its origin, and this effect was linked to the intrinsic spin polarization within the $1 \mathrm{D}$ system by means of transverse electron focusing.

There have been various theoretical proposals on the possibility of nonmagnetic fractional conductance plateaux (below $\mathrm{e}^{2} / \mathrm{h}$ ) in both 2D and 1D systems analogous to the fractional quantum Hall effect (FQHE). ${ }^{13}$ In the FQHE, the fractionalization is principally in the form of odd-denominator fractions as a result of Coulomb interaction among electrons occupying the lowest Landau levels in the 2D system. According to Laughlin's theory and subsequently the Composite Fermion theory, ${ }^{14-17}$ wavefunction antisymmetry results in only odd-denominator fractional states which have been observed. ${ }^{14}$ In addition, an even $5 / 2$ state was found, ${ }^{18}$ and with the advancement in semiconductor growth processes, results were reported where the $1 / 2$ state was observed in bilayer 2D electron gas and in wide, single quantum well systems. ${ }^{19,20}$ In bilayer systems, the presence of two nearby interacting electron layers introduces an additional degree of freedom which possibly allowed the formation of even-denominator fraction at $1 / 2{ }^{21}$ It was also shown that the $1 / 4$ fractional state in the FQHE emerges when 2D electrons were confined in asymmetrical, wide quantum wells. ${ }^{19-22}$

Recently, it was found that in the absence of Landau levels (zero magnetic field), when a quasi-1D system is allowed to reconfigure in a relaxed state such that the $1 \mathrm{D}$ electrons have access to an additional dimension, a variety of fractional conductance states emerged as a function of increasing asymmetry in the confinement potential. The strongest fractional values in units of $\mathrm{e}^{2} / \mathrm{h}$ were $1 / 2,2 / 5$, and $1 / 6$. $^{23,24}$ Although the aspects of this result are similar to the earlier situation where wide and asymmetrical quantum wells resulted in the observation of even-denominator fractional states in the FQHE, the physics of the phenomena is quite different. 
In this letter, we present further evidence that a wide, weakly confined, quasi-1D quantum wire evolves into an unanticipated quantum regime possessing sequential odd-denominator fractional quantum states in the absence of a quantizing magnetic field. We note that such clean flat fractionally quantized plateaux are quite distinct from the mesoscopic conductance fluctuations often found in disordered samples.

The devices used in the present work were fabricated from delta doped GaAs/AlGaAs heterostructures grown using molecular beam epitaxy. The details of various layers are as follows: GaAs cap $(10 \mathrm{~nm}) /$ $\mathrm{Al}_{0.33} \mathrm{Ga}_{0.67}$ As $(200 \mathrm{~nm}) / \mathrm{GaAs}(0.56 \mathrm{~nm}) / \mathrm{As}: \mathrm{Si}\left(8 \times 10^{11} \mathrm{~cm}^{-2}\right) / \mathrm{GaAs}$ $(0.56 \mathrm{~nm}) / \mathrm{Al}_{0.33} \mathrm{Ga}_{0.67} \mathrm{As}(75 \mathrm{~nm}) / \mathrm{GaAs}$ (buffer) $(1000 \mathrm{~nm})$. The twodimensional electron gas (2DEG) formed upto $290 \mathrm{~nm}$ beneath the surface had a low-temperature mobility of $1.6 \times 10^{6} \mathrm{~cm}^{2} / \mathrm{Vs}(2.0$ $\left.\times 10^{6} \mathrm{~cm}^{2} / \mathrm{V} \mathrm{s}\right)$ and an electron density of $9.0 \times 10^{10} \mathrm{~cm}^{-2}(1.0$ $\times 10^{11} \mathrm{~cm}^{-2}$ ) in dark (light). Two samples were fabricated from the same wafer. A pair of split gates of length (L) $0.4 \mu \mathrm{m}$ and width (W) $0.7 \mu \mathrm{m}$ and a top gate of length $1 \mu \mathrm{m}$ separated by a $300 \mathrm{~nm}$ thick insulating layer of cross-linked poly(methyl methacrylate) (PMMA) were patterned by a standard lithographic technique as reported previously. ${ }^{23}$ The two-terminal differential conductance $(G)$ measurements were performed using an excitation voltage of $10 \mu \mathrm{V}$ at $73 \mathrm{~Hz}$ in a cryofree dilution refrigerator with a lattice temperature of $25 \mathrm{mK}$. We avoided illuminating our devices with a red LED in view of the possible role of inhomogeneity in the background potential.

If a mesoscopic system comprises 2D electrons which are confined strongly, then 1D quantized conductance is observed. ${ }^{2-7}$ If the confinement potential is weakened, then a line of electrons can split into two in order to minimize the mutual electron repulsion. This quasi-1D channel is the first step in realizing a zigzag configuration which may be regarded as the onset of Wigner crystallization. ${ }^{6,7}$ It has been shown that the theoretically predicted 1D Wigner crystallization can not only be observed experimentally using the conductance measurement but also be imaged as two separate charge densities using transverse electron focusing. ${ }^{10}$ This phase is very sensitive to the confinement potential and changes in the kinetic energy of the relaxed electrons. One way to modulate the confinement, and hence the wavefunction of weakly confined electrons, is to alter the symmetry of the confinement potential by applying an offset to one of the split gates. The two gates are then swept together, and the offset is then increased for each particular sweep by means of the difference in the voltages applied to each split gate. The basic idea is to achieve an asymmetry in the charge distribution confinement within the 1D channel and investigate its effect on the differential conductance.

Figure 1 shows the results obtained when the confinement potential was varied for each conductance trace as a function of split gate voltage, $\mathrm{V}_{\mathrm{sg}}$, by increasing the asymmetry between the split gates, $\Delta V_{s g}$, at a very low carrier concentration. The top gate voltage, $V_{\mathrm{tg}}$, is kept constant at $-0.53 \mathrm{~V}$, and the initial carrier concentration expressed as a $2 \mathrm{D}$ quantity was $2 \times 10^{10} \mathrm{~cm}^{-2}$. Here, we show the effect produced due to the successive increase in the asymmetry in confinement potential without any magnetic field as shown in (a) and in the presence of an in-plane magnetic field $\left(\mathrm{B}_{\mathrm{II}}\right)$ of $10 \mathrm{~T}$ applied in the plane of the $2 \mathrm{D}$ electron gas and normal to the current direction. The basic idea is to compare the characteristics and determine if they are spin related.

The conductance is shown in units of $\mathrm{e}^{2} / \mathrm{h}$ unless stated otherwise. In Fig. 1(a), the first trace on the right is for a symmetric confinement potential which becomes increasingly asymmetric on going toward the left. Considering the behavior from right (symmetric) to left (asymmetric), initially, there is no structure [apart from $3 / 2$, i.e., $\left.0.7\left(2 \mathrm{e}^{2} / \mathrm{h}\right)\right]$ and the conductance increases smoothly with split gate voltage. As the asymmetry and width of the $1 \mathrm{D}$ channel increase, a plateau forms close to 1 (trace shown in black), indicating that the system has spontaneously spin polarized. This plateau then becomes unstable and decreases in value until it becomes stabilized at $2 / 3$ before disappearing at the maximum asymmetry which appears to be a
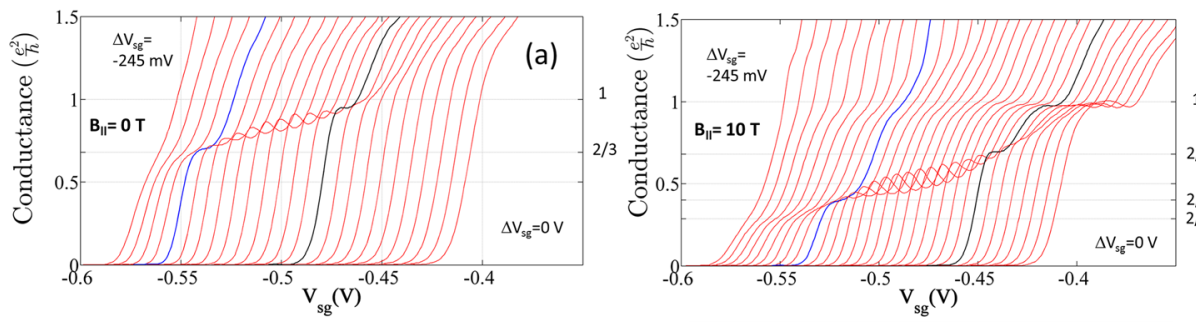

(b)
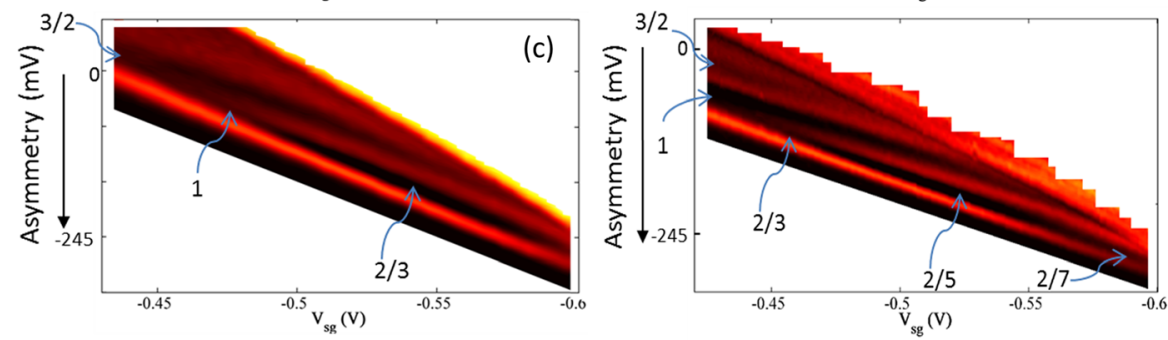

(d)

FIG. 1. Effect of enhancing the asymmetry in the confinement potential on the differential conductance (sample 1). (a) At $B_{\|}=0 \mathrm{~T}$, plateaux at 1 and $2 / 3$ were observed as shown in black and blue traces, respectively, as a function of $\Delta \mathrm{V}_{\mathrm{sg}}\left(0 \mathrm{~V}\right.$, right, symmetric potential) to $-245 \mathrm{mV}$ (left) in steps of $-10 \mathrm{mV} ; \mathrm{V}_{\mathrm{tg}}=-0.53 \mathrm{~V}$. A shoulder corresponding to $3 / 2$ [or $0.7\left(2 \mathrm{e}^{2} / \mathrm{h}\right)$ ] is seen at $\Delta \mathrm{V}_{\mathrm{sg}}=0 \mathrm{~V}$. (b) At $\mathrm{B}_{\|}=10 \mathrm{~T}$, the spin polarized 1 is strengthened (first trace on the right, symmetric potential). The previous 1 [observed in (a) in black trace] now becomes $2 / 3$, and the previous $2 / 3$ [observed in (a) in blue trace] is now $2 / 5$ shown in a blue trace. (c) and (d) The color plots of transconductance of data shown in (a) and (b), respectively. Here, the red region represents risers and the black region represents plateaux in the conductance plot. 
featureless transition to 2D transport [Figs. 1(a) and 1(c)]. The plateau at 1 , the last trace on the extreme left, has disappeared leaving just the $3 / 2$ state. The results presented here provide further evidence of the emergence of nonmagnetic fractional states in low-density weakly confined quantum wires. ${ }^{23}$ The color plots in (c) and (d) show the transconductance of data shown in (a) and (b), respectively.

In Fig. 1(b), due to the presence of the large in-plane magnetic field of $10 \mathrm{~T}$, the expected spin polarized plateau is observed at 1 , and with increasing asymmetry and weaker confinement, an additional plateau is also formed at $2 / 3$. Moving to the left, the $2 / 3$ plateau drops and becomes unstable until a stable, plateau forms at $2 / 5$; this plateau then decreases in value to form a structure near $2 / 7$ before all features disappear in a similar manner to Fig. 1(a). The results imply that the magnetic field has enhanced the formation of $2 / 5$ and $2 / 7$ although $2 / 3$ is always present and is clearly spin polarized at $B_{I I}=0 \mathrm{~T}$ [Fig. 1(d)]. A comparison of the gate voltages in Figs. 1(a) and 1(b) for plateau formation is not straightforward due to the magnetic field induced diamagnetic shift. ${ }^{10}$

Figure 2 illustrates the sensitivity of the system as a function of carrier density at a fixed in-plane magnetic field of $11 \mathrm{~T}$. There is a constant offset on the split gate voltage producing the asymmetry of confinement $\left(\Delta V_{s g}=-180 \mathrm{mV}\right)$, and the top gate produces a decreasing carrier concentration from left to right, which is expressed as a $2 \mathrm{D}$ quantity, and this varies between $6.0 \times 10^{10} \mathrm{~cm}^{-2}$ and $1.0 \times 10^{10} \mathrm{~cm}^{-2}$. On the extreme left, at $\mathrm{V}_{\mathrm{tg}}=-0.34 \mathrm{~V}$, a spin polarized plateau is formed due to the presence of a large in-plane magnetic field. On reducing the carrier concentration at $\mathrm{V}_{\mathrm{tg}}=-0.47 \mathrm{~V}$, a plateau appears at 2/5. It appears as if a series may be attempting to form in that in addition to $2 / 5$, the most stable values are found at or near $1 / 4,1 / 8$, and $1 / 10$ and a weak state at

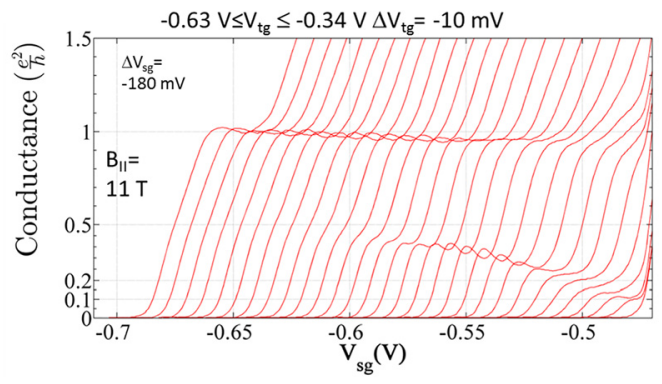

(a)

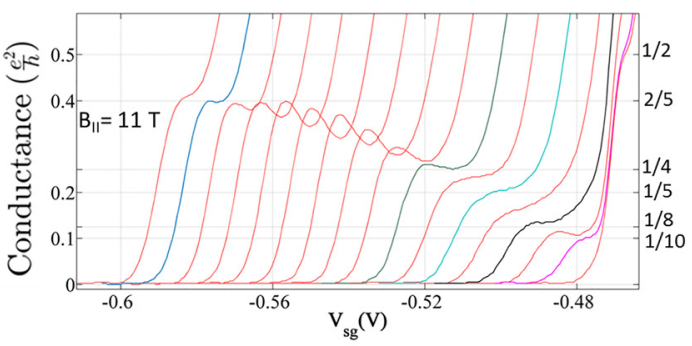

FIG. 2. Effect of the top gate variation on the fractional states in the presence of $B_{\|}$ (sample 2). (a) The top gate was varied from $-0.34 \mathrm{~V}$ (left, strongest confinement) to $-0.63 \mathrm{~V}$ (right, weakest) in steps of $-10 \mathrm{mV}$, at $\mathrm{B}_{\|}=11 \mathrm{~T} ; \Delta \mathrm{V}_{\mathrm{sg}}=-180 \mathrm{mV}$. On reducing the carrier concentration, a fractional state emerged at $2 / 5$ followed by a series of resonating structures terminating at $1 / 4$. Flat plateaux were then observed at $1 / 8$ and $1 / 10$. (b) The plot shows a magnified view of the data shown in (a).
$1 / 5$. Decreasing the carrier concentration results in a region of instability between $2 / 5$ and $1 / 4$, and there is only weak evidence of a state at $1 / 2$ [trace in magenta on the right, Fig. 2(b)] just before the transition to 2D. It may be noted that the usual spin polarized state at 1 is present in all traces [Fig. 2(a)]. We note that the slope of the $2 / 5$ plateau successively becomes negative and then settles and almost flattens on reaching the 1/4 state. Generally, a flat or wider plateau signifies full transmission, i.e., a signature of a $1 \mathrm{D}$ band. We note that the structure at $1 / 10$ is accompanied by a structure at $1 / 2$ in the weakest and lowest 2D density of $\sim 1.0 \times 10^{10} \mathrm{~cm}^{-2}$, probably suggesting that they are hybrid states. It has been reported that the $1 / 2$ and $1 / 4$ fractional states in the FQHE are enhanced when electrons are confined in a wide, asymmetric quantum well; however, in a symmetric charge distribution, these fractions do not appear. ${ }^{25}$ It is suggested that they could be Pfaffian wavefunction. ${ }^{26}$

Figure 3 shows the effect of temperature on the 2/5 conductance plateau measured in sample 2 in the absence of a magnetic field. It may be noted that $2 / 5$ remains almost stable up to $300 \mathrm{mK}$, and beyond this temperature, it broadens and eventually smears out. The experimental result is in agreement with the theoretical simulation by Shavit and Oreg. ${ }^{27}$ The result also shows that a true band is present which exhibits thermal broadening similar to typical 1D bands. At the lowest temperature, the plateau loses flatness which may be due to the number of carriers decreasing as $\mathrm{k}_{\mathrm{B}} \mathrm{T}$, where $\mathrm{k}_{\mathrm{B}}$ is the Boltzmann constant and $\mathrm{T}$ is the operating temperature and unable to backscatter and form a cyclic current between the legs of the zig-zag. Within the experimental limits and considering uncertainty in the carrier concentration due to its confinement dependent, we estimated the low temperature range for the observation of the $2 / 5$ fractional state using $T \ll\left(\frac{u}{L}\right) \cong T_{L}$, where $u$ is the Luttinger parameter and an $\mathrm{L}$ is the $1 \mathrm{D}$ channel length, to be in the range of $0.1-0.5 \mathrm{~K}$, which is reasonably in agreement with the results.

The presence of a large in-plane magnetic field in addition to electron-electron interaction may result in a more complex pattern of cyclic motion. ${ }^{23}$ Although the zig-zag is only present in the plane of the electron gas, there may be an antiphase correlated motion normal to the plane to reduce the repulsion which possibly drives the fractionalization. In addition, when the carrier concentration is low, it seems most likely that the even-denominator values arise from all the electrons in the channel, forming a collective state involving pairing. Our results show the nonmagnetic fractional states dependent on the asymmetry of charge distribution of the electron gas within a quasi-1D

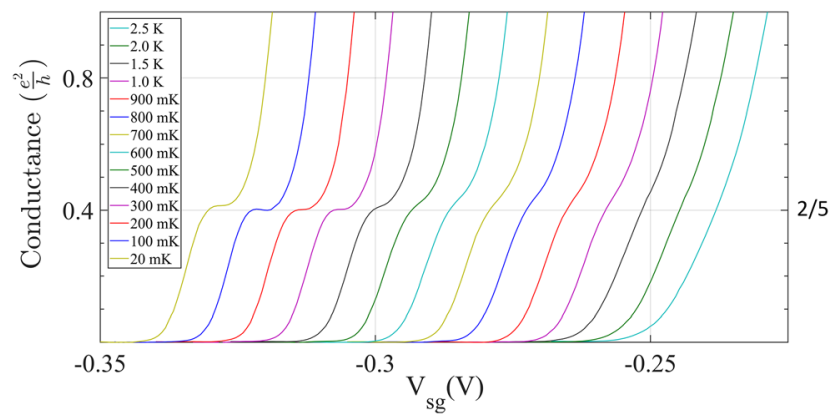

FIG. 3. Temperature dependence of the $2 / 5$ fractional state observed in the absence of a magnetic field; $\mathrm{V}_{\mathrm{tg}}=-0.36 \mathrm{~V}$ and $\Delta \mathrm{V}_{\mathrm{sg}}=90 \mathrm{mV}$ (sample 2, second cooldown). Traces have been offset horizontally for clarity. 
channel. A complete understanding of such results requires further detailed investigation both experimentally and theoretically.

To summarize, the interactions between the electrons create correlated motion and fractional behavior emerges in the absence of Landau levels, giving rise to fractional conductance plateaux, and the results may indicate the start of a series varying as $1 /(2 n+1)$, where $n$ is an integer.

We thank Professor S. Bose and Professor K. Berggren for many fruitful discussions. The work was funded by the Engineering and Physical Sciences Research Council (EPSRC), United Kingdom (Reference: EP/R029075/1). One of us (S.K.) is grateful to the United Kingdom Research and Innovation (UKRI) for a Future Leaders Fellowship (Reference: MR/S015728/1).

\section{REFERENCES}

${ }^{1}$ T. Thornton, M. Pepper, H. Ahmed, D. Andrews, and G. Davies, Phys. Rev. Lett. 56, 1198 (1986)

${ }^{2}$ D. A. Wharam, T. J. Thornton, R. Newbury, M. Pepper, H. Ahmed, J. E. F. Frost, D. G. Hasko, D. C. Peacock, D. A. Ritchie, and G. A. C. Jones, J. Phys. C 21, L209 (1988)

${ }^{3}$ B. Van Wees, H. Van Houten, C. Beenakker, J. G. Williamson, L. Kouwenhoven, D. Van der Marel, and C. Foxon, Phys. Rev. Lett. 60, 848 (1988).

${ }^{4}$ K. J. Thomas, J. T. Nicholls, M. Y. Simmons, M. Pepper, D. R. Mace, and D. A. Ritchie, Phys. Rev. Lett. 77, 135 (1996).

${ }^{5}$ W. Hew, K. Thomas, M. Pepper, I. Farrer, D. Anderson, G. Jones, and D. Ritchie, Phys. Rev. Lett. 101, 036801 (2008).

${ }^{6}$ W. Hew, K. Thomas, M. Pepper, I. Farrer, D. Anderson, G. Jones, and D. Ritchie, Phys. Rev. Lett. 102, 056804 (2009); L. W. Smith, W. K. Hew, K. J. Thomas, M. Pepper, I. Farrer, D. Anderson, G. A. C. Jones, and D. A. Ritchie, Phys. Rev. B 80, 041306(R) (2009).
${ }^{7}$ S. Kumar, K. J. Thomas, L. W. Smith, M. Pepper, G. L. Creeth, I. Farrer, D. Ritchie, G. Jones, and J. Griffiths, Phys. Rev. B 90, 201304(R) (2014).

${ }^{8}$ J. S. Meyer and K. Matveev, J. Phys.: Condens. Matter 21, 023203 (2008).

${ }^{9}$ A. Klironomos, J. S. Meyer, T. Hikihara, and K. Matveev, Phys. Rev. B 76, 075302 (2007).

${ }^{10}$ S.-C. Ho, H.-J. Chang, C.-H. Chang, S.-T. Lo, G. Creeth, S. Kumar, I. Farrer, D. Ritchie, J. Griffiths, G. Jones, M. Pepper, and T.-M. Chen, Phys. Rev. Lett. 121, 106801 (2018).

${ }^{11}$ C. Yan, S. Kumar, K. J. Thomas, P. See, I. Farrer, D. Ritchie, J. Griffiths, G. Jones, and M. Pepper, J. Phys.: Condens. Matter 30, 08LT01 (2018).

${ }^{12}$ C. Yan, S. Kumar, K. J. Thomas, P. See, I. Farrer, D. Ritchie, J. Griffiths, G. Jones, and M. Pepper, Phys. Rev. Lett. 120, 137701 (2018).

${ }^{13}$ B. I. Halperin, Phys. Rev. B 25, 2185 (1982).

${ }^{14}$ R. B. Laughlin, Phys. Rev. Lett. 50, 1395 (1983).

${ }^{15}$ J. Jain, Adv. Phys. 41, 105 (1992).

${ }^{16}$ D. C. Tsui, H. L. Stormer, and A. C. Gossard, Phys. Rev. Lett. 48, 1559 (1982).

${ }^{17}$ R. Roy and S. L. Sondhi, Physics 4, 3 (2011).

${ }^{18}$ R. Willett, Rep. Prog. Phys. 76, 076501 (2013).

${ }^{19}$ Y. W. Suen, L. W. Engel, M. B. Santos, M. Shayegan, and D. C. Tsui, Phys. Rev. Lett. 68, 1379 (1992).

${ }^{20}$ Y. W. Suen, H. C. Manoharan, X. Ying, M. B. Santos, and M. Shayegan, Phys. Rev. Lett. 72, 3405 (1994).

${ }^{21}$ J. Shabani, T. Gokmen, Y. T. Chiu, and M. Shayegan, Phys. Rev. Lett. 103, 256802 (2009).

${ }^{22}$ D. R. Luhman, W. Pan, D. C. Tsui, L. N. Pfeiffer, K. W. Baldwin, and K. W. West, Phys. Rev. Lett. 101, 266804 (2008).

${ }^{23}$ S. Kumar, M. Pepper, S. N. Holmes, H. Montagu, Y. Gul, D. A. Ritchie, and I. Farrer, Phys. Rev. Lett. 122, 086803 (2019).

${ }^{24}$ Y. Gul, S. Holmes, M. Myronov, S. Kumar, and M. Pepper, J. Phys.: Condens. Matter 30, 09LT01 (2018).

${ }^{25}$ J. Shabani, T. Gokmen, and M. Shayegan, Phys. Rev. Lett. 103, 046805 (2009).

${ }^{26}$ G. Moore and N. Read, Nucl. Phys. B 360, 362 (1991).

${ }^{27}$ G. Shavit and Y. Oreg, Phys. Rev. Lett. 123, 036803 (2019). 